\title{
Childhood obesity: Socio-cultural determinants
}

\author{
*V P Wickramasinghe ${ }^{1}$
}

Sri Lanka Journal of Child Health, 2018; 47(3): 193-203

DOI: http://dx.doi.org/10.4038/sljch.v47i3.8538

(Key words: Childhood obesity, socio-cultural determinants)

The President and the Council of the Sri Lanka College of Paediatricians, Past Presidents, Fellows and Members of the Sri Lanka College of Paediatricians, Members of the family of the late Dr. Stella De Silva, Distinguished Invitees. I take this opportunity to thank you and your Council for giving me this opportunity to deliver this prestigious oration this year. Ladies and Gentlemen, I stand here in all humility to pay a regal tribute to a dazzling star, as described by Dr. B.J.C. Perera in the Inaugural Memorial Oration, a star in the medical scenario in the bygone days of this small island state, which has excelled in health care.

Stella Gertrude de Silva was born on the $2^{\text {nd }}$ of June 1918 in Randombe, Balapitiya in the Southern province of Sri Lanka, as the fourth child in a family of six to Mr. C.R. De Silva, a well-known lawyer, and Mrs. Adeline de Silva. She began her studies from Prajapathy Vidyalaya, a local school in Ambalangoda and later continued at Southlands College, Galle. Young Stella had a strong ambition to study medicine one day, which was a rare feat in those days. Although science subjects were not offered at Southlands College at that time, with the help of her teachers, she was able to study them at Richmond College, Galle. After an illustrious all round career at Southlands College, at the tender age of 19 years, teenage Stella entered the Ceylon Medical College in 1937. In 1942, she qualified, fulfilling her childhood ambition. In 1952, the Government of Ceylon awarded her a scholarship to be trained in paediatrics for two year in the United Kingdom. In November 1954, she returned to the country and was appointed to the Lady Ridgeway Hospital. In 1959, she was appointed as a Consultant Paediatrician to the very same hospital, as well as the maternity hospitals in the group, till her retirement in 1973.

She received many honours, and in 1994 the Sri Lankan Government bestowed on her the title Vidyajyothi. In 1985 she set up the Paediatric

\footnotetext{
${ }^{1}$ Professor in Paediatrics, University of Colombo

*Correspondence: pujithaw@yahoo.com

The author declares that there are no conflicts of interest

Personal funding was used for the project.

Open Access Article published under the Creative

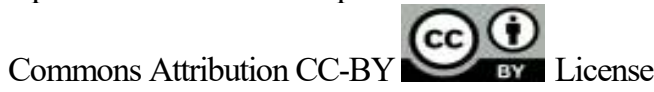

Department at the North Colombo Medical College and was the Founder Professor. After an illustrious career, on the $12^{\text {th }}$ of April 2012, at the age of 93, she closed her eyes forever, leaving behind a legacy of work and a galaxy of medical trainees to serve this country and carry the flame she lit. Although I had never met this great personality, it gives me great pleasure to stand here in all humility to deliver the fifth Dr. Stella de Silva Memorial Oration to this august audience on this morning of the $15^{\text {th }}$ of December 2017.

\section{Introduction}

Health generally refers to a state of wellbeing and in 1948 the World Health Organization definition shows that it is not merely absence of disease but a physical, mental, social and spiritual wellbeing ${ }^{1}$. Health is largely determined by the socio-economic, cultural and environmental conditions in which we live ${ }^{2}$. Obesity is a multi-dimensional phenomenon resulting from an interaction between medical and social spheres $^{3}$. The economic cost of non-communicable diseases (NCDs) is very high. According to the WHO 2014 country profile for Sri Lanka, 75\% of deaths in Sri Lanka are due to $\mathrm{NCDs}^{4}$. However, the distressing fact is that $18 \%$ of the population is at risk of dying of 4 NCDs between 30-70 years of age, which is the most productive time of a person's life. This is only the tip of the iceberg, and there are many more with NCDs seeking treatment which leads to a huge burden on the economy at both micro and macro levels. Furthermore, this could even adversely affect the high life expectancy that we have achieved as a nation. Prof. Paul Zimmet, former Director of the International Diabetes Federation, once predicted that this could be the first generation which could die before their parents ${ }^{5}$.

Obesity, one of the primary risk factors for NCDs, is spreading in epidemic proportions all over the world, even in places where undernutrition is not yet controlled, and it is fast penetrating into the paediatric population. In 2015,108 million children and 604 million adults worldwide were estimated to be obese and the majority were from Asia ${ }^{6}$. During the last 35 years obesity has doubled in more than 70 countries while showing a rise in other parts as well. The rate of increase in childhood obesity is higher than that in adult obesity ${ }^{6}$. Obesity is a major public health problem resulting in adverse socio-economic, political, physical and psychological effects on both the individual and the society. Childhood obesity is a 
multi-dimensional public health problem now affecting all geographic areas whether urban or rural and all economies of developed as well as developing countries. Many countries face the double burden of malnutrition and a national survey conducted by the Medical Research Institute (MRI) of Sri Lanka and published by Dr. Renuka Jayatissa et al has clearly shown that. In that study, among the 10-15 year old age group, the overall prevalence of stunting was $28.5 \%$ and underweight $47.2 \%$, with $2.2 \%$ being overweight and obese ${ }^{7}$.

With timely interventions undernutrition can be treated effectively. However, despite all forms of treatment modalities available, correcting childhood obesity is not easy. Therefore, primary prevention is important and crucial. In 2016, the WHO came up with a Commission Report on Ending Childhood Obesity, the ECHO Report ${ }^{2}$, and this was supplemented by an Action Plan in 2017 which addresses 6 main domains to act upon, namely intake of healthy food, physical activity, pre-conception and pregnancy care, early childhood diet and physical activity, health promotion at school and weight management ${ }^{8}$. The action framework for ending childhood obesity outlines the importance of giving correct leadership on managing the interventions in order to achieve intermediate and long-term outcomes. In Sri Lanka, the College of Paediatricians should take a greater initiative in implementing the recommendations of the $\mathrm{ECHO}$ report.

Obesity develops mainly due to energy imbalance in the background of genetic predisposition. However, many socio-economic and cultural factors beyond simple nutriomics contribute to the development of obesity, thereby leading to the development of many metabolic complications.

Sri Lanka progressed from a low-income country in 1960 s with a GDP of less than US\$ 250 to a level closer to US\$ 4000 by 2015 . Parallel to the socioeconomic development, the life expectancy had increased gradually from about 55 years in 1950s to almost 75 years by 2015 with reduction in malnutrition, infant mortality and low birth weight. One of the main contributors to the improvement of health indices, apart from the socio-economic improvement, is the high female literacy rate, which was more than $90 \%$ by 2010 . All these changes have contributed to the improvement in the health and wellbeing of the Sri Lankans. The rapid socioeconomic, demographic and nutritional transition has resulted in changes in dietary habits and lifestyles leading to new health problems such as overweight and obesity leading to an increased risk of NCDs. Therefore, health professionals, media, politicians and society as a whole, have made a significant focus on the problem.

No phenomenological research designs have been done before to uncover the effects of individual, family and society on childhood obesity. A conceptual framework was developed to understand the socio-cultural and environmental factors that influence the development of obesity in 5-12 year old children (Figure 1). This will also uncover the subjective realities of parents' perception of children's fatness and their psycho-social issues, focused on an urban setting of Sri Lanka.

\section{Conceptual Framework}

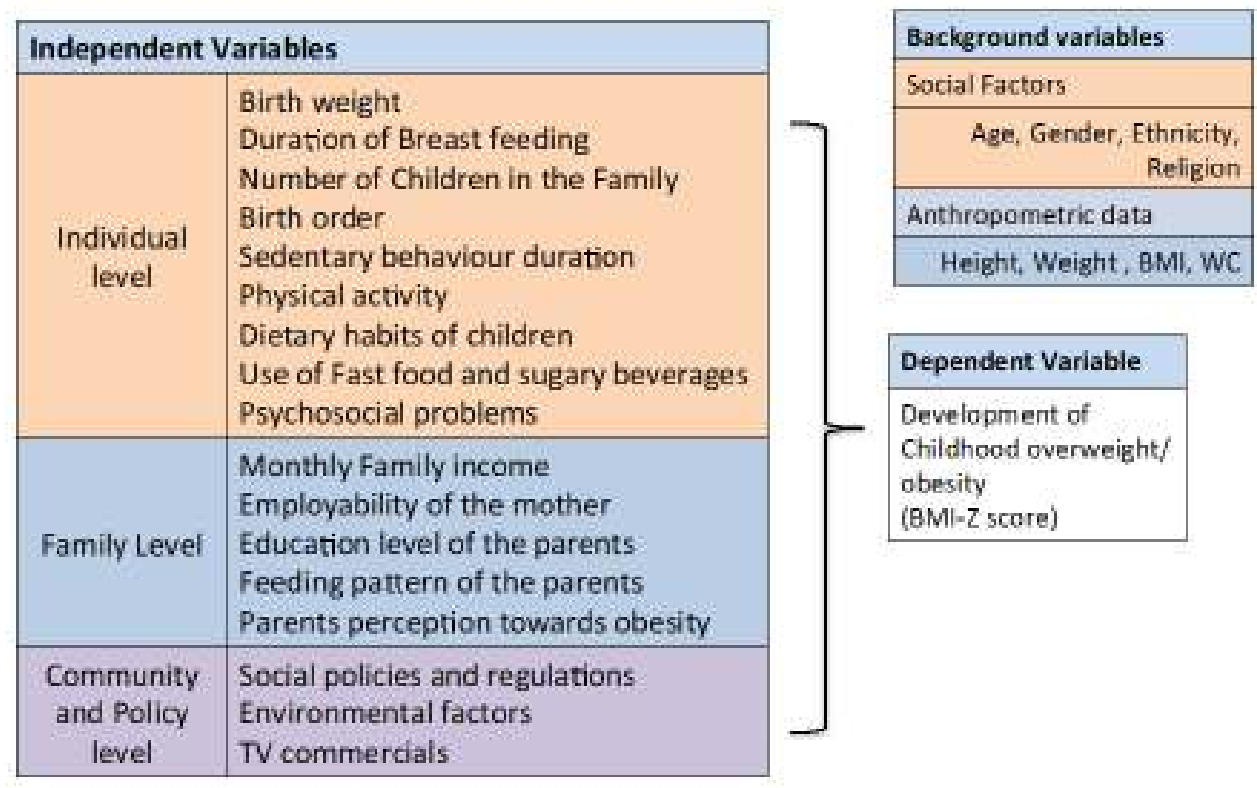

Figure 1: A conceptual framework developed to understand the socio-cultural and environmental factors that influence the development of obesity in children 
In the Sri Lankan context, obesity is considered as a state of 'beauty', a 'symbol of prosperity' and a manifestation of 'healthiness'. Most of the studies on obesity have looked at the medical aspect, but not at the socio-economic, environmental, behavioural and psychological factors that would affect the family and child. Obesity is a socially constructed lifestyle disease seen in modern society. Understanding the influence of socio-cultural factors would help to understand the roots of this illness. The Social Ecological Theory, which is the interaction of the individual and family within the social, institutional and cultural contexts that make up the state of wellbeing, would help to understand the roots of this condition. It represents the child in the centre with different societal levels of influencers surrounding in an ascending manner (Figure 2).

\section{Social Ecological Model}

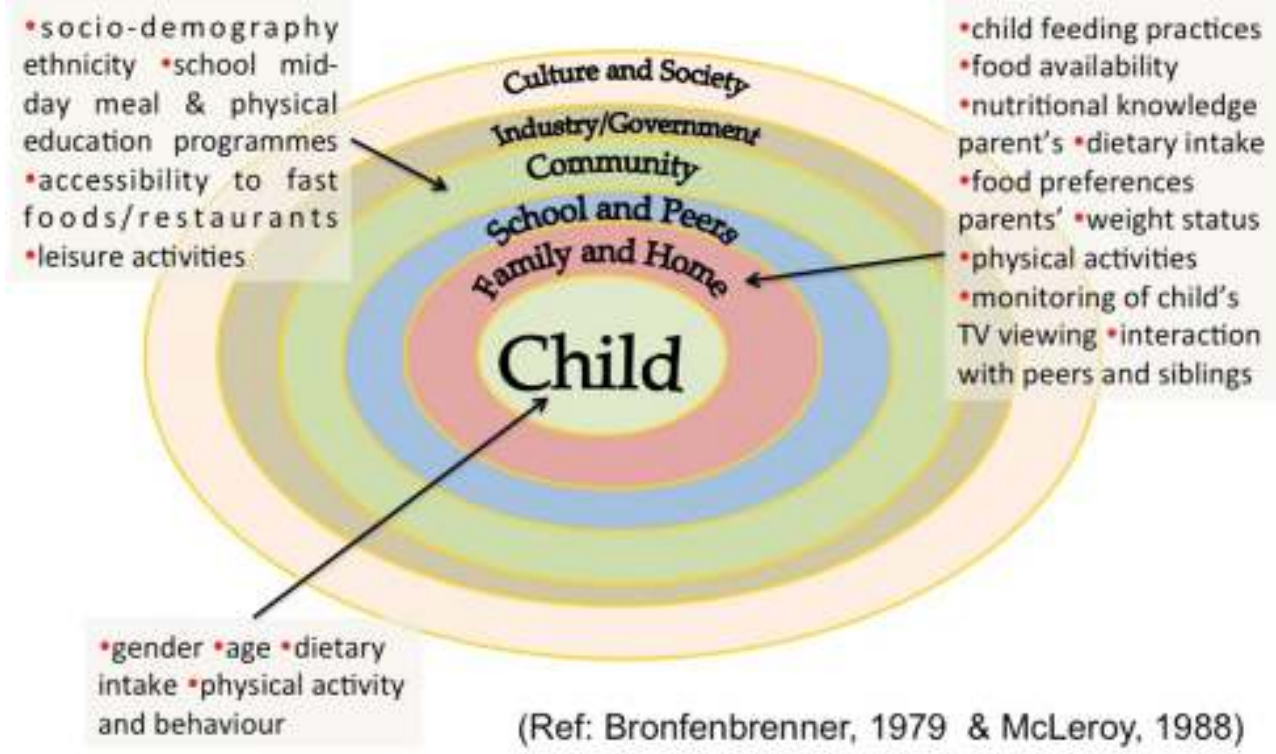

Figure 2: Social Ecological Model, representing the child in the centre with different societal levels of influencers in an ascending manner

\section{Method}

A descriptive cross sectional study with a comparison group was conducted adopting qualitative and quantitative data collection techniques in normal and obese 5-12 year old children and their parents. Obese children were recruited from the Nutrition Clinic of the Lady Ridgeway Hospital (LRH) for Children, Colombo, while normal children were selected from those attending five schools within the Colombo Municipality. One hundred obese children were consecutively recruited from the clinic and 200 children using cluster sampling technique from five schools. Ethics Review Committees of the University of Colombo and Lady Ridgeway Hospital for Children approved the protocol.

Quantitative and qualitative data were collected using pre-tested interviewer-administered questionnaire, semi-structured interviews, focus group discussions (FGDs) with both children and parents, pile sorting, drawings, case studies and basic anthropometric measures. Three FGDs with ten parents each of both obese and non-obese children and case reports of children, covering areas of food consumption, facing competitive examination and daily routines, perception towards obesity, barriers to its prevention, and psychological issues including stigma were evaluated. Anthropological drawing was used to collect data focused on children's food preferences, TV characters, food outlets visited, how they were bullied and their psycho-social issues.

\section{Data analysis}

Quantitative data were analysed using descriptive methods and qualitative data using thematic analysis method where there was familiarization, generating initial codes, searching themes among codes, reviewing data and defining and naming themes and interpretation. All statistical processes were conducted using SPSS - 20.0 Version for Windows.

\section{Results \\ Demographic and socio-economic characteristics of children}

The study population consisted of 300, 5-12 year old children, recruited from the obesity clinic at LRH $(n=100)$ and from schools in the Colombo municipal council $(n=200)$. There were $181(60 \%)$ boys and 119 $(40 \%)$ girls. The sample consisted of $156(52 \%)$ obese children and 144 (48\%) non-obese children (Table 1). 
Table 1: Demographic and socio-economic characteristics of the study population

\begin{tabular}{|l|r|r|}
\hline \multicolumn{1}{|c|}{ Characteristic } & $\begin{array}{c}\text { Children in obese group } \\
(\boldsymbol{n = 1 5 6 )}\end{array}$ & $\begin{array}{c}\text { Children in non-obese group } \\
(\boldsymbol{n}=\mathbf{1 4 4})\end{array}$ \\
\hline Gender: Number (\%) & $109(69.9)$ & $72(50)$ \\
Male & $47(30.1)$ & $72(50)$ \\
Female & $09.3(02.0)$ & $08.9(02.0)$ \\
\hline Average age in years: Mean (SD) & $23.2(03.4)$ & $14.5(02.0)$ \\
\hline Body mass index $\left(\mathrm{kg} / \mathrm{m}^{2}\right)$ : Mean (SD) & $02.3(0.74)$ & $-01.4(01.4)$ \\
\hline Body mass index - Z score: Mean (SD) & $03.3(0.7)$ & $03.2(0.7)$ \\
\hline Birth weight in kg: Mean (SD) & $04.7(01.17)$ & $04.9(01.2)$ \\
\hline No. of family members: Mean (SD) & $58,012.8(63,852.1)$ & $38,826(32,963.9)$ \\
\hline Family income (Rupees/month): Mean (SD) & & \\
\hline
\end{tabular}

The mean ages of both groups were similar. The obese group was taller, heavier and had a higher BMI than their non-obese counterparts. The average household size was 4.8 persons and it is on par with the national average household size of 4.3. Statistically significantly higher monthly household income was seen in the obese group compared to non-obese counterparts.
Overweight increased with the increase in the family income with $62 \%$ of the non-obese children being in the lower income category (Figure 3), which was comparable to a previous study conducted within the locality ${ }^{9}$.

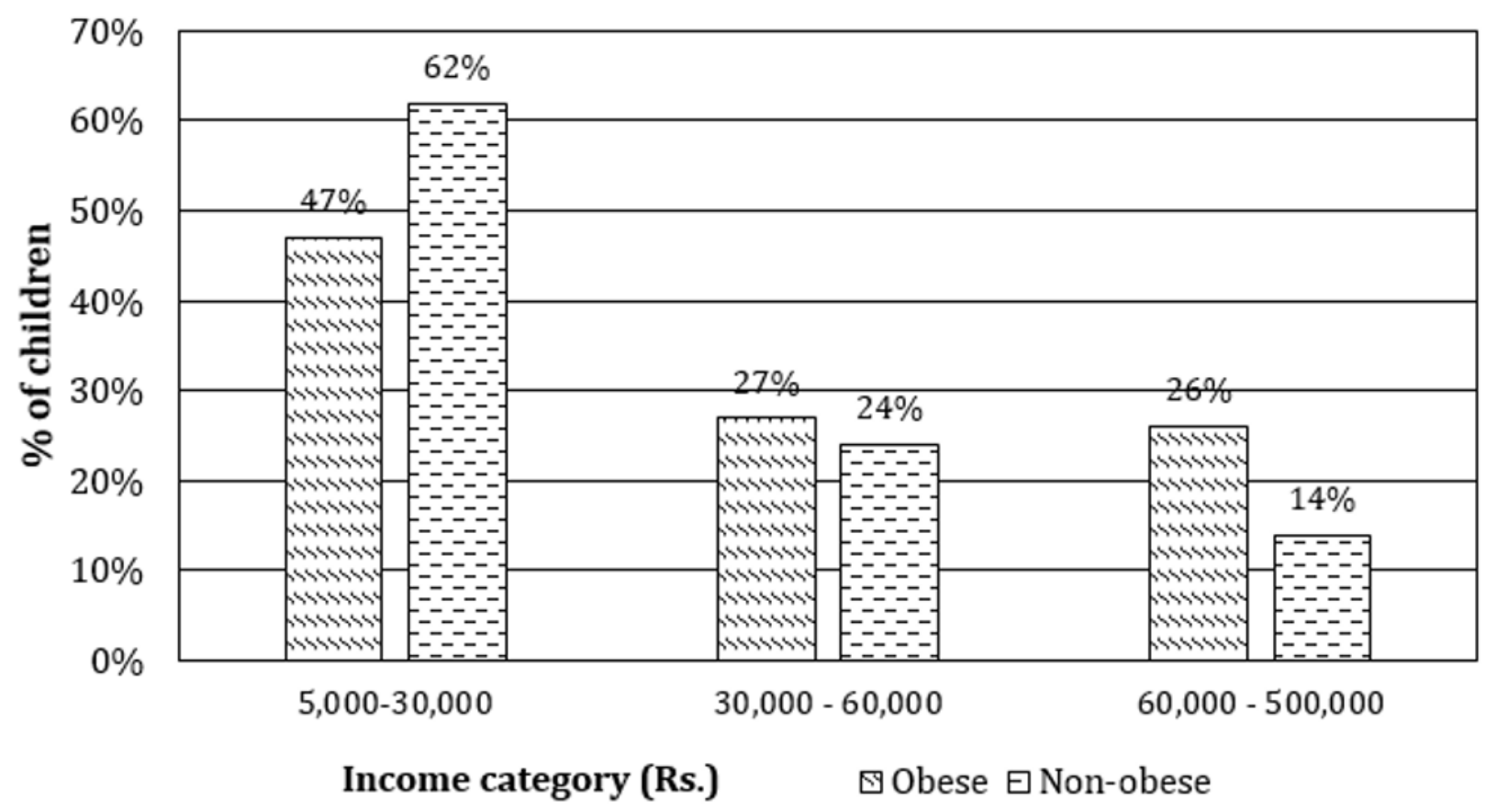

Figure 3: Distribution of Nutritional status according to income category

In order to identify the socio-cultural determinants of childhood obesity, qualitative and quantitative data were categorized into different themes based on the Social Ecological Approach (Figure 2).

1. Individual level or child factors

2. Family \& Home level factors
3. School Environment and Peer factors

4. Industry, Government, Culture and Society

\section{Individual}

Association between birth weight and BMI-Z score is shown in figure 4. 

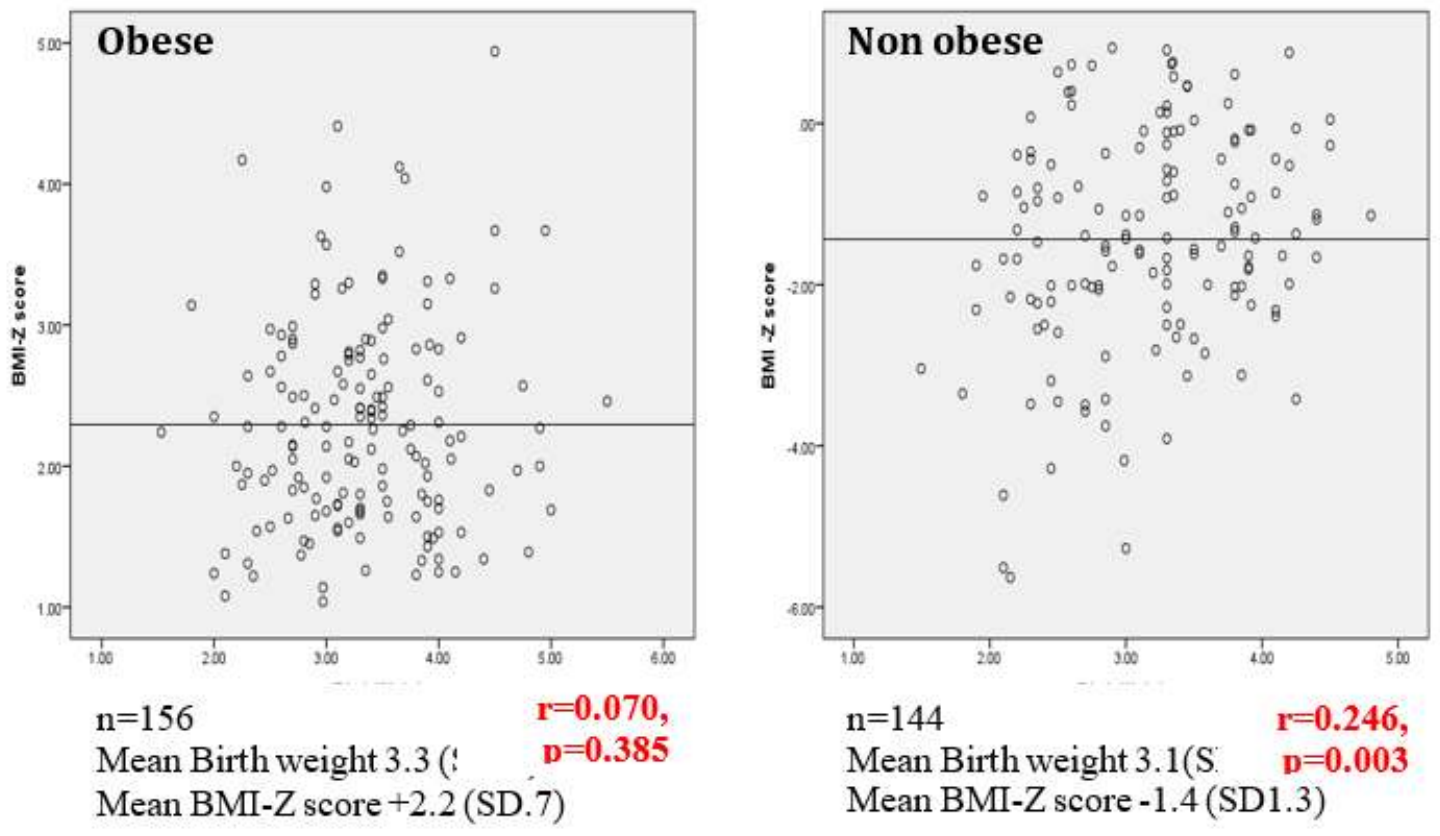

Figure 4: The relationship between birth weight and state of obesity

The relationship between birth weight and BMI-Z score in non-obese children, showed significant positive correlation $(\mathrm{r}=0.246, \mathrm{p}=0.003)$ but there was no significant association seen in the obese population $(\mathrm{r}=0.070, \mathrm{p}=0.385)$. It could be inferred that non-obese children had a healthy growth matching their birth weight with their current weight, but obesity would have been a result of influences of environmental factors independent of the inherent growth potential, thus accumulating fat due to inappropriate feeding and lack of physical activity.

The distribution of nutritional status according to gender and age is shown in Figure 5.

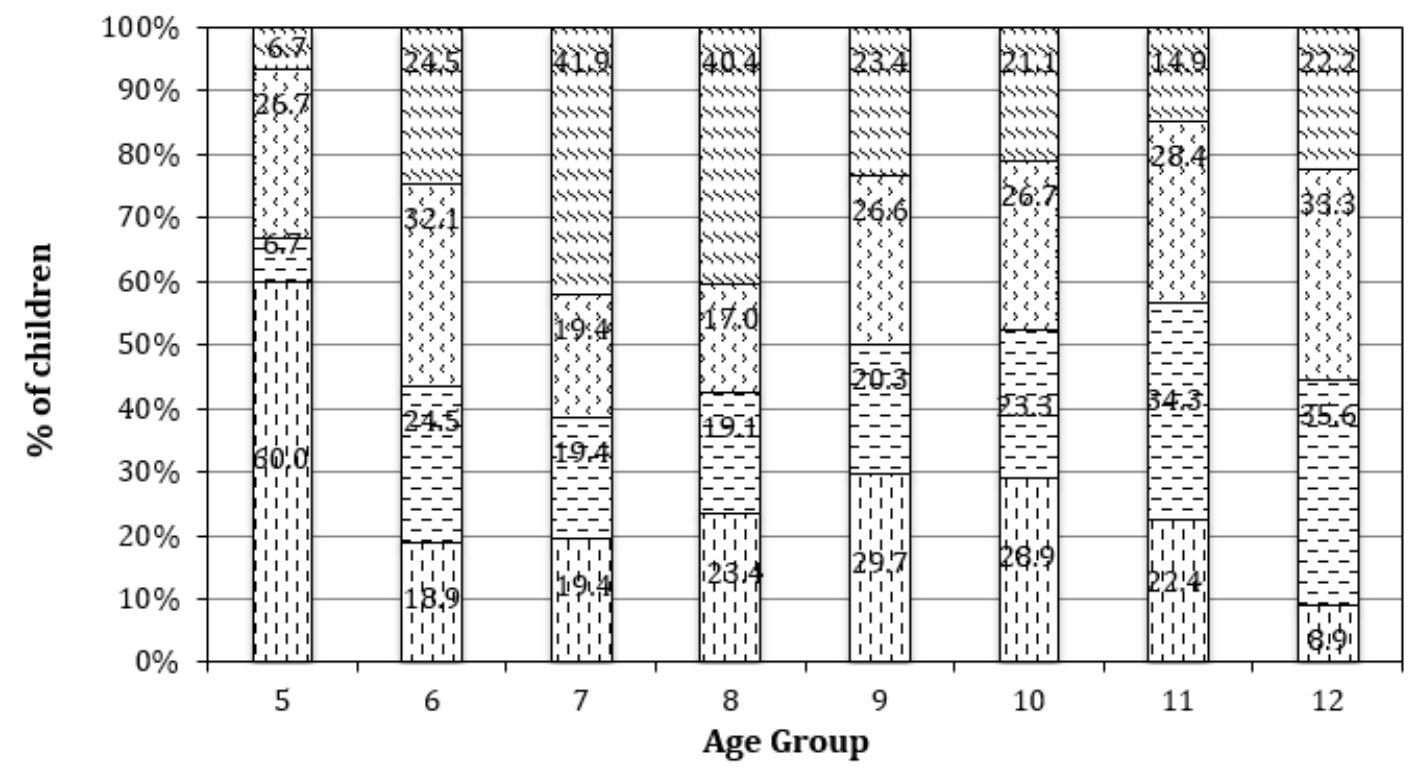

$\nabla$ Non-obese boys $\square$ Non-obese girls $\square$ Obese boys $\square$ Obese girls

Figure 5: Distribution of the nutritional status according to gender and age.

From six years onwards there is a rise in the percentage having obesity with more boys being affected than girls. Differences to the pattern seen in 5 and 12 years are probably due to imbalance in the proportions recruited from the nutrition clinic.

\section{Food consumption patterns}

Food is a primary need of a person and is influenced by culture, beliefs \& values, socioeconomic status and environment. As previous studies have explained, examining the food consumption patterns is important to understand the determinants of childhood obesity. 
Majority of non-obese children had regular meal patterns in both gender groups (boys $75 \%$ and girls $81 \%$ ). However, only $42 \%$ of girls and $52 \%$ of boys in the obese category showed regular meal patterns. Further, the comparison of food consumption patterns according to age in the two categories revealed that the non-obese had regular meal patterns almost throughout all age categories (data available but not shown). However in the obese, the irregular meal patterns gradually increase with age, probably increasing independence contributing to their feeding habits. Obese children consumed food that promotes obesity such as fried chicken, junk food and soft drinks more frequently.

\section{Parental perception on child's feeding}

The quantity and the frequency of intake of food were highlighted by these narratives by mothers.

"We can't count even how many times he eats. Normally, before going to the school he eats rice or drinks milk with more than 5 biscuits. In the school and on the way home, he eats whatever he likes.
After returning from school he eats rice while watching $T V$, and even after a bath, he used to eat rice or a small packet of biscuits and in the night too he eats a large portion of rice".

\section{Sedentary behaviour and screen time}

Two most influential inventions that contributed to sedentary behaviour are the Chair and Screen devices. Humans did not evolve to sit and function, but chairs made them spend most of the day seated. Chairs enabled people to sit for long hours and engage in sedentary behaviour without much effort and they could lose insight on how much of time they had been spending on sedentary activities. TV viewing was the main sedentary life activity identified and it was high among obese children, followed by academic work at home (Figure 6). Regular attendance of after school private classes, playing video games and sleeping in the daytime were the other risk factors identified. A similar pattern of behaviour was observed in a previous study $^{10}$.



Mean duration (Hrs) घobese $\square$ Non-obese

\section{Figure 6: Distribution of types of sedentary behavior according to nutritional status}

As a habit, almost $96 \%$ of the children of the study population used to watch $\mathrm{TV}$ while having their meals at home. Almost $80 \%$ of obese children viewed TV for more than 15 hours a week. Little over $40 \%$ of the non-obese children viewed TV more than 15 hours a week. Compared with non-obese children, these differences were significant.

Parents described the magnitude of the problem, showing how some children get addicted to screen devices.
- "My son gets ready by 5.30 am. Till the school van comes he watches cartoon. In the afternoon also, he watches cartoon from $3.30-5.30 \mathrm{pm}$ until his evening private tuition teacher comes" (a mother)

- "He is addicted to cartoons. Even though friends call him to play, he doesn't want to go. He doesn't get a chance to be with his friends. He is an isolated child spending time by the side of the TV" (A mother). 
A little over a third of this sample played video games, probably limited by affordability. Twelve year old children reported playing video games for a longer duration than others. Five year old children in this sample did not engage in video games. However, with the increase in smart phone use these could change rapidly.

\section{Educational activity}

Students spent a lot of time on academic activities, which should be the norm to a certain extent. However, the main driver is the grade five scholarship examination that makes them attend more extra classes each week. Overall, the obese child spent more time attending private classes than a nonobese child and it showed a clear positive relationship to the family income. Children from Government national schools and from International /private schools attended more private classes than children from Government non-national schools. However, these differences were not statistically significant. Children as young as 5 years attended extra classes, but by 10 years (grade 5) $85 \%$ of the study group attended extra classes for 15 hours or more per week.

The following narrations showed the social pressures faced by children and how it may have impacted their life style. Further, it showed how parents have given priority to education over health. This social phenomenon clearly exhibited the situation of changing social priorities in the society with the socio-economic transitions.

- "Daily we have to do one hour sample test paper targeted for the scholarship exam before school begins. After school and at home, I have to do all the extra academic work for the day."

"My mother insists that I should finish homework of the day before going to bed" (A girl).

- "I know he has a problem with his weight. But, we are waiting till his scholarship exam is over" (A mother).

\section{Physical activity}

In 2010 WHO put out Global guidelines for physical activity for 5-17 year old children where 60 minutes of moderate to vigorous daily physical activity was recommended. Sleep had been identified as an important integral part of daily activity and the American Academy of Paediatrics has put out a guideline on the duration of sleep for children of different ages ${ }^{11}$.

The descriptive statistics showed that the mean hours of engaging in physical activity by obese and nonobese children were 4.1 and 6.1 hours per week respectively. Almost $40 \%$ of obese children did not engage in any vigorous physical activity. There was no significant difference between genders. At the same time, children attending private schools spend less time on physical activity and most of these schools did not have adequate space. Therefore it is important to place minimum standards/requirements for physical activity space before establishment of private schools to ensure adequate physical activity.

These narrations show how children are addicted to screens and how they find the liberty to use them and reluctance to engage in physical activity.

- "I like IGG, car race games and YCD games. We have to pay only Rs. 100 per hour. Most of the time we play phone games, laptop games or computer games." (A boy).

- “Once I find my uncle's or aunt's mobile phone I play games. I like games such as Superman, Panda, Construction and Harry Porter. Is not like playing cricket or other game in the ground, we can do it within a short time." (A boy).

- "I always prefer to be the umpire during outdoor games because I can't run fast” (A boy).

- "We like to play 'hand cricket' in the school. It is so easy and enjoyable. It is very interesting and easy to play while being inside the class" (A boy).

However there are also constraints faced by children as well as parents. Issues related to lack of space and unsafe neighbourhood is growing each day. Even if they want to engage in physical activity these issues restrict it.

- "I like cycling very much. But, my mother is scared to send me out on to the road. I don't have enough space in the garden to play" (A boy).

- "We are in the staff quarters... My husband is abroad. I'm afraid to go alone with children in the walking path. So, he bought an 'Orbi Track' for us. But, children were interested only for a few days. Now it has just become a 'cloth rack' (A mother).

Obese children also do face many socioenvironmental issues. They are highlighted in these case reports. One main social issue is that they cannot choose clothes of their desire.

- There are no clothes for me in the kids' section. Normally, my mother stitch clothes for me. But, I like to wear as my friends do. I can only wear stretching materials and once I wear such clothes they are tight" (A girl).

- "It is not easy to find a suitable trouser for my son. I'm compelled to go to more than ten shops to buy one denim trouser for him." (A mother of a 5 year old child).

\section{Family and home}

\section{Feeding practices of the children}

"My son was born with a normal weight but his sister was above average. However, later, she became leaner. So, I decided not let that happen to my son. I 
kept on feeding him with lot of food; baby rusks, eggs, cereals, cheese, potato every day. As the TV advertisement says I use 'Pedia Pro' (milk powder) to develop his intelligence" (A mother).

FGD showed how mothers were eager feeding their children and what they expected; "if it is a boy he should be like a tusker" and the volume of food they need to give; "A boy needs seven pots of milk" and feeding frequency; "A boy feels hungry seven times within a time in which a Tamarind leave takes to fall".

Sometimes the parents are also helpless with the influence of the extended family.

- "It is difficult to control the food intake of the children with their grandparents. When we are too strict on food intake, grandparents feel sorry and they give money or buy foods for children. (A mother).

Birth order could play a part on the nutritional status of the child. Forty nine percent of the obese children were first born. This could probably be due to enthusiastic feeding as well as availability of time with diversion of a higher proportion of resources on food.

Frequency of feeding out of home is shown in Figure 7.

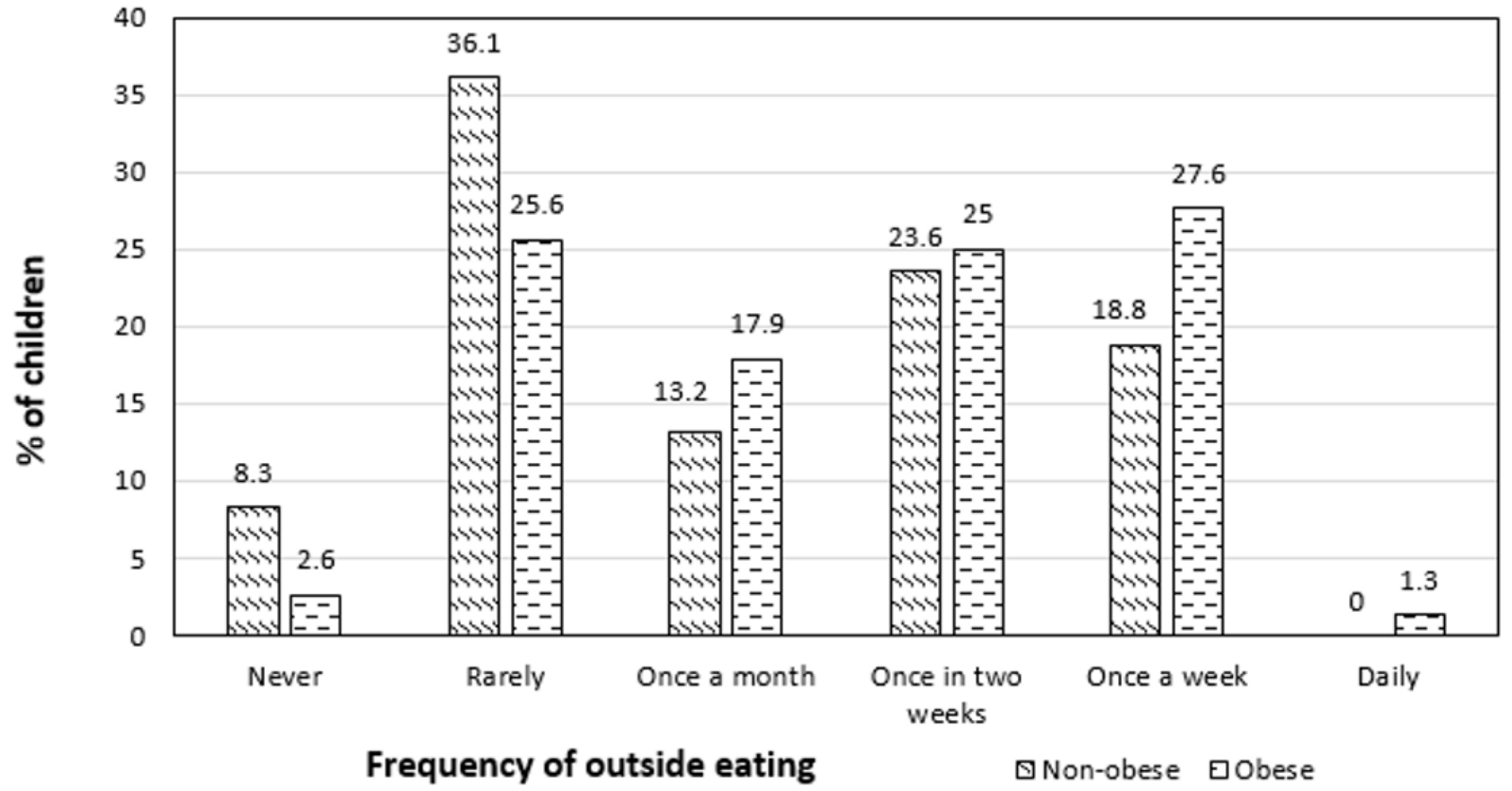

Figure 7: Distribution of frequency of feeding outside home according to nutritional status

More obese children were seen among those who were eating out of home more frequently. Practices built by parents cultivate the habit in children as well. Parents' eating habits influence children's feeding habits as most of the behaviour in children develops from imitation.

- "The child is like the father. He demands spicy foods. After all, he takes a big portion. Sometimes, he suggests going out for eating as his father does. Now, father is in Italy. So, I have to take him out for meals" (A mother).

\section{Physical activity}

The household belief and culture has an effect on the opportunity to engage in physical activity. Opportunities given to girls are much less and it would have an impact not only on obesity but also on conditions such as osteoporosis later in life.
- 'In our culture, we don't allow girls to go for outdoor physical activities alone. And also, girls have more issues with safety. Due to our cultural restrictions, we as women, have been confined to the house." (A mother -Muslim).

- "Daughters are not like sons, they have to engage in household work. But, boys when they are at home they only watch TV or play games on computer or phone." (A mother).

The parent-child behaviour interaction has an impact on the development of obesity. Surrendering to child's demands to overcome social embarrassment is a major factor that contributes to childhood obesity. 


\section{Parental perception towards child's adiposity and psycho-social issues}

In Sri Lankan culture people love to have a chubby child and consider it with pride. Due to lack of knowledge on its impact on long-term health, people are not worried and even conditions like acanthosis is considered as a simple skin condition.

- "We love chubby children. In fact, she is a pride to the family. Children with slender bodies are easy victims of illnesses. Though chubby children fall ill they recover quickly" (A mother).

- "We don't consider fatness as a problem, because everything is fine with them. Sometimes, we are worried only about their skin. Since there are some black patches behind the neck and it must be a small skin problem" (A mother).

However, with more public awareness, people tend to worry about being overweight, as it affects both physical health and the psycho-social state. However, many think that it would get corrected with time as they grow, while some who want to seek clarification, are unaware where to get help from as well as how urgently they should do so. My experience is that many do not still seek help for obesity per se. Even in this series, more than $60 \%$ were referred by the OPD when they visited for some other medical condition. Only a mere $7 \%$ brought their child to hospital seeking help for obesity.

To combat obesity, we should get to the root causes. Parents, who are the main carers, should be aware of these causes and they identified the following as the lead cause. Although many parents identified the problem, it was difficult for them to execute a management plan to treat and control obesity due to lack of knowledge on the condition, lack of support from the extended family, time and space constraints. Many get frustrated in this process and wish they could have a medication.

- "She tells the grandfather that I am not giving her food. They (grandparents) blame me. They give chicken without asking me. But, I'm the one who is being blamed for her fatness. It is difficult to get support from the others in the family. So I get depressed." (A mother)

\section{School environment and peers}

The school is one of the major obesogenic environments. Children of this age group from schools of higher socio-economic (SE) strata have higher levels of obesity while wasting and stunting is more prevalent in schools with a low SE background. Similar data were seen from a study in 2004 where in private schools in Colombo, the prevalence of obesity/overweight was highest ${ }^{9}$. The environment, as well as the social class of those who attend different levels of schools, would have an impact.

\section{Availability of food at school}

Many children buy food from the school canteen and it is more prevalent among children from higher SE strata and also in private and national schools. Most of these items are high in sugar, salt and oil content. Impact of the school canteen on a child's life is quite evident. If canteens could provide 'healthier' food items, children would not refuse to take home prepared meals. However, as long as school canteens function as profit generating ventures, this practice would continue.

"Not like in the past, the school canteens have everything. Sometimes, though I prepared meals my son refuses to take food to school as buying foods from the canteen is easier. There are a lot of unhealthy foods. But, we can't prevent what he eats during the school time". (A mother)

\section{Physical activity at school}

Health and Physical education subject provides the necessary education emphasizing the importance of proper feeding and physical activity on prevention of NCDs. Textbooks are prepared in all 3 languages in a basic form also providing information as extra reading material for parents.

However, lack of qualified teachers, inadequate basic facilities, and moreover utilization of the allocated time for other subjects, prevent children from maximally utilizing the opportunity.

Although the school environment is thought to be safe, it may not be the case and parents have a lot of concerns in letting children participate in activities that would otherwise encourage physical activity.

- "We have heard that, among the school cadets, there are some misconduct happening and they use chubby children mostly for such misconduct." 'So, I'm afraid of sending him for cadetting though it would be helpful to reduce the weight. The particular room is also far from the office premises. Nobody is there to supervise even." (A mother).

\section{Bullying}

Children who are overweight/obese face many issues, either physical, social or psychological. Some of the nicknames used by peers to tease them, cannot be tolerated. Individuals at different levels in society, family, relatives and peers subject these obese children to psychological abuse. Some remarks could be even highly personal targeted at their physical state or body parts. Most of them do not have coping strategies and find it hard to overcome these.

\section{Industry, government, culture and society Promotion campaigns}

Food advertisements are a major influencer for developing childhood obesity. As we know today, the food industry is a multi-million, multi-national 
industry driven to generate profit. Fierce competition exists between different chains. Therefore they use large sums of money to advertise, trying to attract more and more customers. Children, who lack the ability to rationalize. fall prey. About a quarter of the sample was attracted to the advertisement itself and another similar number to the appearance of the food.

The advertisers use many catchy phrases, which register in their minds and even become a part of day-to-day language. Children depicted in their drawings influences the advertisements made on them. Also, when they drew the type and portion sizes, there was a clear distinction between the obese and non-obese group's feeding habits. Larger portion sizes of more 'non nutritious' food were the obese children's choice.

Some cartoon characters influenced their eating behaviour and also nicknames were generated by peers based on such characters.

Marketing agencies carefully select their actors. The personalities promoting them attract the public initially. Subsequently, substances like MSG, salt and sugar make the children get addicted. Furthermore, some of the advertisements promote the use of webbased games which contribute to the promotion of sedentary behaviour and use of screen devices for recreation.

Most probably the persons who appear on these advertisements may not be aware of the harm that they could cause, and I think we, the professionals, also have a responsibility to address it publicly, for at least then they would be able to take an informed decision before contracting to the advertisement.

Marketing agencies also attract children and parents to food items by offering complementary toys and other material. Legislature needs to be formulated at least for the food industry to stop such promotions. The introduction of the new food act would pave the way in the near future prohibiting targeting children under 12 years of age.

Most of the cultural events are being commercialized and stakeholders related to food industry always make offers, which are attractive but are harmful to health. Parents were concerned about the number of food advertisements aired during the cartoon programmes. Most of the popular television channels, during their evening cartoon times, had allocated a quarter of the airtime for advertisements and almost a third to half of the advertisements are on food and beverages.

Although parents may want to buy more nutritious food, it may not be easy due to unattractiveness and prohibitive prices. However, most of the time junk food is attractive and cost-effective, especially with commercial promotions.
It is important that policy makers do address such issues by offering subsidies for cultivation and introducing effective ways of storing and transportation of food thus helping to maintain good texture and reduce post-harvest losses thus reducing the price and improving the quality when it reaches the end consumer.

Although the school canteen circular was out in 2007 there are a lot of difficulties in implementing it. Till recently, each day all canteens had to contribute a sum of money in rupees equal to the student population of the school to the school development fund. This drove canteen owners to generate sufficient profit at the end of each day and they were compelled to sell food attractive to children. However, in 2015 through deliberations with the Ministry of Education (author was part of the group) managed to abolish that requirement.

\section{Conclusions}

Childhood overweight/obesity, is a result of socioeconomic transformation. The factors uncovered through qualitative data revealed that lack of space, time, motivation to play and deliberate ignorance of the physical exercises at the school are some of the main contributory factors. In particular, within the school setup, changing social priorities from health to education has also influenced. Paying much attention to competitive education system by parents too has led children to follow the rat race in education. According to their schedule of the day, the priority has been given totally to education and not even a small consideration for physical activities.

Prof. Hettige, a Professor in Sociology of the University of Colombo had illustrated this phenomenon quite eloquently. "More and more people appear to be resorting to consumption as a way of demonstrating their relative position in the social hierarchy. Unlike the nationally rooted middle class, very much a product of the state sector, the members of the new rich class do not derive their social status and prestige from the positions they occupy in society, but by demonstrating living in modern houses and apartments, owning luxury vehicles and other status symbols, overseas travels, educating children in private international schools, shopping at modern supermarkets, eating out at expensive restaurants, adoption of western food habits etc. have become the defining features of life of the metropolitan elite. With rising incomes, the adoption of these consumption patterns has not been difficult at all for the emerging, transnationally oriented business elite."12

Therefore enough evidence had been unfolded to show that in today's context, childhood obesity in Sri Lanka is a problem of socio-economic transformation without adequate empowerment of society. The psychological cost, financial cost and impact on longterm health is immense. 


\section{Recommendations}

More than at micro level, issues at macro level are immense. As part of control of overweight/obesity, strengthening proper early feeding and monitoring, highlighting the importance of proper feeding and physical activity for children and adolescence, strengthening advertising and marketing regulations, creating safe schools and neighbourhoods with adequate recreational facilities are some of the areas that need addressing.

Mr. President, ladies and gentlemen, the Paediatrician community in the past took immense effort to introduce programmes to control communicable diseases and under-nutrition, of which the fruits of success are now being enjoyed. However, I don't think we can be complacent and relax, as a new challenge is emerging which needs urgent action and I hope the SLCP would be able to take the leadership as most of the interventions have to happen at policy planning level.

\section{Acknowledgements}

I thank Ms. Priyadarshani Premarathne, Dr. Dulani Samaranayake, Professor Amarasiri de Silva and Dr. Mithila Mendis for being behind the designing and carrying out the different sections of work presented here. I also thank all children and their parents for volunteering to participate in the study. Last but not least I thank all of you for your patient listening and accepting our invitation to be here in spite of your busy schedules.

\section{References}

1. World Health Organization. Basic Documents. Geneva: WHO; 2014.

2. World Health Organization. Report on Commission Report on Ending Childhood Obesity. Geneva: WHO; 2016.

3. World Health Organization. Global status report on noncommunicable diseases 2010. Geneva: WHO; 2011.

4. World Health Organization. World Health Organization - Noncommunicable Diseases (NCD) Country Profiles, 2014. Geneva: WHO; 2014.

5. Federation ID. The IDF Consensus definition of the Metabolic Syndrome in Children and Adolescents. Brussels: International Diabetes Federation; 2007.
6. Collaborators GO, Afshin A, Forouzanfar MH, Reitsma MB, Sur P, Estep K, et al. Health Effects of Overweight and Obesity in 195 Countries over 25 Years. New England Journal of Medicine 2017; 377(1):13-27. https://doi.org/10.1056/NEJMoa1614362 PMid: 28604169 PMCid: PMC5477817

7. Jayatissa R, Ranbanda RM. Prevalence of challenging nutritional problems among adolescents in Sri Lanka. Food and Nutrition Bulletin 2006; 27(2):153-60. https://doi.org/10.1177/15648265060270020 6

PMid: 16786981

8. World Health Organization. Report of the Commission on Ending Childhood Obesity: implementation plan: executive summary. Geneva: WHO; 2017.

9. Wickramasinghe VP, Lamabadusuriya, S.P., Atapattu, N., Sathyadas, G., Kuruparanantha, S., Karunarathne, P.. Dietary and physical activity patterns of school children in an urban area of Sri Lanka. Sri Lanka Journal of Child Health 2005;34(2):6.

10. Perera U, Abeysena, C. Prevalence of overweight and obesity in grade five students in schools of Gampaha Medical Officer of Health area. Journal of the Community of Physicians of Sri Lanka 2014;19(2):6.

https://doi.org/10.4038/jccpsl.v19i2.7576

11. Paruthi S, Brooks LJ, D'Ambrosio C, Hall WA, Kotagal S, Lloyd RM, et al. Recommended Amount of Sleep for Pediatric Populations: A Consensus Statement of the American Academy of Sleep Medicine. Journal of Clinical Sleep Medicine 2016; 12(6):785-6.

https://doi.org/10.5664/jcsm.5866

PMid: 27250809 PMCid: PMC4877308

12. Hettige S. Transformation of society, In: Lakshman WD, Tisdell CA, editors. Sri Lanka's development since Independence: Socio-economic perspectives and analyses. New York, Nova Science Publishers Inc.: 2000. 19-39 